\title{
NOVAS TECNOLOGIAS, NOVAS MÍDIAS, VELHAS \\ DIFICULDADES: aprimorando a interface com o usuário para a escolha de base de dados ou periódicos \\ NEW TECHNOLOGIES, NEW MEDIAS, OLD DIFICULTIES:
}

improving the user interface to choose a research database or periodical

\author{
Ana Cristina de Freitas Griebler - agrieble@etcom.ufrgs.br \\ Mestre em Gerência da Informação pela North Carolina Central University, USA \\ Bibliotecária de Referência na Biblioteca Setorial da Escola Técnica da UFRGS \\ Professora Substituta do Curso Técnico em Biblioteconomia da mesma Escola \\ Ana Maria Mattos - ammattos@ea.ufrgs.br \\ Especialista em Gestão Universitária pela UFRGS \\ Bibliotecária na Biblioteca Setorial da Escola de Administração \\ Mestranda no Programa de Pós-Graduação em Ciência da Informação \\ Escola de Ciência da Informação da UFMG
}

\begin{abstract}
Resumo
As tecnologias da informação e comunicação determinaram profundas transformações no modo pelo qual transitam, no meio acadêmico, as publicações e outras modalidades de informações científicas. Com o desenvolvimento destas tecnologias, os veículos utilizados pelos pesquisadores para a realização de suas investigações passaram a ser bastante diversificados. O periódico publicado na forma impressa passou a compartilhar seu espaço com o periódico disponibilizado por via eletrônica. A grande quantidade de informações disponíveis trouxe dificuldades em sua recuperação, tanto para os pesquisadores quanto para os bibliotecários, suscitando dúvidas: Como aprimorar a interface disponibilizada ao usuário para a escolha da base de dados a ser consultada? Em qual base de dados pode ser encontrado o título do periódico desejado? Este título periódico é indexado em mais de uma base? Este periódico está disponível em texto completo ou somente através de seu resumo? Qual base de dados deve ser consultada sobre um tema específico? Bibliotecários de universidades norte-americanas propuseram soluções para estes problemas, visando facilitar e otimizar a busca destas informações e sua vinculação com a coleção descrita nos catálogos das bibliotecas. $\mathrm{O}$ presente trabalho propõe a aplicação da proposta norte-americana, em uma versão adaptada à realidade brasileira para os usuários das bibliotecas da Universidade Federal do Rio Grande do Sul (UFRGS). Mais especificamente, se oferece uma interface que pode integrar automática e simultaneamente a pesquisa do usuário para o sistema de bibliotecas da UFRGS, o conjunto de bases de dados e de periódicos do Portal Capes, e para as bases gratuitas disponíveis na Internet.
\end{abstract}

Palavras-chave: Biblioteca universitária. Recuperação da informação. Pesquisa bibliográfica.

\section{ORIGEM E PANORAMA ATUAL: NOVAS TECNOLOGIAS, NOVAS MÍDIAS}

O periódico científico surgiu em Paris, no ano de 1665. O francês Denis de Sallo editou o Journal de Sçavans que informava sobre livros publicados na Europa, resumindo seus Enc. Bibli: R. Eletr. Bibliotecon. Ci. Inf., Florianópolis, n. 23, 1º sem. 2007. 
conteúdos, divulgando experiências em Física, Química e Anatomia, entre outros assuntos. Logo em seguida, em março do mesmo ano, um grupo de filósofos ingleses, ligados à Royal Society, edita o Philosophical Transaction - periódico publicado regularmente, que se propunha a divulgar as correspondências trocadas entre os membros da sociedade e seus colegas europeus (MEADOWS, 1999). Passados mais de 300 anos do nascimento do modelo tradicional do periódico científico, a evolução da ciência, o modelo de promoção adotado nas carreiras universitárias e os critérios de concessão de verbas para fomento à pesquisa incrementaram o crescimento exponencial da publicação de revistas científicas, fazendo surgir alguns problemas: (a) demora na publicação do artigo; (b) alto custo de aquisição e manutenção das compras destas publicações impressas; e (c) ineficiência dos instrumentos de pesquisa para a localização de um assunto, dentre as múltiplas opções de periódicos publicados (MUELLER, 2000). Em face destes problemas práticos, foi iniciada a busca de alternativas para solucionar estas dificuldades:

[...] o meio eletrônico foi vislumbrado como a esperança da solução há muito buscada, já que oferece mais rapidez na comunicação e flexibilidade de acesso, tem largo alcance e baixo custo relativo, disponibilidade imediata, é capaz de diminuir a necessidade de manutenção de coleções, barateando os custos (MUELLER, 2000, p. 81, grifo nosso).

No mundo inteiro, o desenvolvimento tecnológico ocorrido nos meios de comunicação fez do periódico eletrônico uma ferramenta indispensável para os pesquisadores.

Bibliografias especializadas surgiram na Europa, no final do Século XV e no início do Século XVI. Porém, foi no Século XIX, com a intensificação do mercado editorial dos periódicos científicos, que a necessidade de como e onde encontrar a informação procurada passou a ser uma questão imperativa. Para atender a esta demanda, surgiram os periódicos de indexação e os resumos, objetivando facilitar o acesso à informação dispersa em diferentes publicações, reunindo-as e publicando-as em uma lista dos trabalhos produzidos sobre determinado assunto ou área de conhecimento, permitindo assim, a identificação de artigos de periódicos e outros trabalhos. "As primeiras áreas cobertas pelos periódicos de indexação e resumos foram as ciências básicas e aplicadas, tais como a Química, Engenharia, Zoologia e Medicina" (CENDÓN, 2000, p. 219). Na década de 1970, diferentes empresas comerciais começam a produzir periódicos de indexação e resumo, e outras áreas do conhecimento (Ciências Sociais e Humanas) começam a ser indexadas.

O desenvolvimento da tecnologia dos computadores, na década de 60, permitiu inovações nas formas como os serviços de indexação e resumo atendiam sua clientela. [...] os serviços passaram a produzir e comercializar os seus índices em forma de bases de dados. Hoje, muitos dos serviços de indexação e resumos produzem índices, 
tanto em forma impressa como em versão eletrônica [...]. Inicialmente disponíveis apenas para consulta local, já na década de 60, as bases de dados puderam ser acessadas remotamente, via redes de computadores de comutação de pacotes. A partir de 1985, passaram também a ser produzidas e disseminadas utilizando a tecnologia de CD-ROM. A difusão da Internet, na década de 90, facilitou ainda mais o acesso remoto às bases de dados (CENDÓN, 2000, p. 220-221, grifo nosso).

Atualmente, existem muitos tipos de bases de dados (de resumos, de citações, de sumários, etc.), produzidas por diversos tipos de organizações (grandes empresas, associações profissionais, órgãos governamentais, editoras comerciais, etc.) buscando atender a todos os tipos de necessidade informacional que qualquer pesquisador possa ter (CENDÓN, 2000). A base de dados eletrônica tornou-se uma ferramenta fundamental para que o pesquisador desempenhe suas atividades de investigação. Paradoxalmente, a quantidade de bases de dados que existem atualmente, a exemplo das publicações periódicas, torna a busca da informação desejada uma tarefa árdua e demorada. Passam a ser comuns as situações em que os pesquisadores se perguntam: “Afinal, em qual base devo pesquisar?", ou "Qual o período de cobertura desta base?", e “Ela indexa que tipo de publicação?”. É necessário encontrar respostas rápidas e eficientes para questões tão elementares quanto estas.

A Internet foi criada em 1969, patrocinada pelo Departamento de Defesa Norte Americano, objetivando permitir o compartilhamento de computadores entre os diferentes pesquisadores norte-americanos que trabalhavam em projetos militares. No Brasil, o início da conexão às redes internacionais foi dado pelo Laboratório Nacional de Computação Científica (LNCC) ao conectar-se com a University of Maryland, em 1988. No mesmo ano, a Fundação de Amparo à Pesquisa do Estado de São Paulo (FAPESP) conectou-se com o Fermi National Laboratory, em Chicago. No ano seguinte, a Universidade Federal do Rio de Janeiro (UFRJ) se conectou à University of California at Los Angeles, por intermédio da rede Bitnet. $\mathrm{O}$ objetivo era a comunicação com pesquisadores de outras universidades e centros de pesquisa, localizados no exterior. Em 1989, foi implementada a Rede Nacional de Pesquisa, formando a espinha dorsal da rede de computadores. O principal objetivo destas conexões foi o de promover e incentivar o intercâmbio de informações entre cientistas brasileiros e estrangeiros (TEIXEIRA; SCHIEL, 1997). Presentemente, a Internet faz parte do cotidiano do ensino, pesquisa e extensão das universidades brasileiras.

As novas tecnologias de informação e comunicação (TICs), juntamente com o crescimento exponencial do mercado editorial de periódicos e bases de dados, fomentaram a criação de uma rede com muitas informações. Porém, a desordem com a qual a informação está sendo disponibilizada traz elevados custos de busca e, portanto, a abundância informacional pode levar ao desperdício de tempo e de recursos, tanto 
financeiros quanto humanos e materiais. Como os bibliotecários podem contribuir para otimizar este processo? É preciso facilitar o uso de toda esta estrutura de comunicação.

\section{NOVAS TECNOLOGIAS, NOVAS MÍDIAS, VELHAS DIFICULDADES}

As TICs determinaram profundas transformações no modo pelo qual transitam, no meio acadêmico, as publicações e outras modalidades de informações científicas. Com o desenvolvimento destas tecnologias, os veículos utilizados pelos pesquisadores para a realização de suas investigações passaram a ser bastante diversificados. A grande quantidade de informações disponíveis trouxe dificuldades em sua recuperação, tanto para os pesquisadores quanto para os bibliotecários, suscitando muitas dúvidas. Como aprimorar a interface com o usuário para facilitar a escolha da base de dados ou periódico a ser consultado? Paim e Nehmy (1998) observam que:

A avaliação de sistemas de informação, em razão da crescente disponibilidade de dados possibilitada pelas novas tecnologias, torna-se hoje um dos temas mais relevantes para profissionais e pesquisadores desse campo de interesse. A maior facilidade, tanto de provimento quanto de acesso, desencadeia novos problemas com os quais os praticantes da área se deparam ao propor desenhos ou avaliar o uso de sistemas de informação [...] (PAIM; NEHMY, 1998, grifo nosso).

Com o desenvolvimento das TICs, o comportamento dos usuários também foi modificado. Eles se "auto-atendem" em casa, na sala de aula, no laboratório de informática, na sala do pesquisador/professor. A biblioteca funciona, de certo modo, em tempo integral, e sua localização e horário de atendimento já não são mais tão fundamentais para a obtenção de determinado tipo de publicação. Entretanto, tornou-se imperativo melhorar a interface de acesso do usuário à coleção de bases de dados e de periódicos de que a biblioteca dispõe, sejam estas impressas ou eletrônicas, pagas ou gratuitas na Internet, ajudando a aumentar o êxito nesta busca da informação desejada.

Este "auto-atendimento" é entendido por Fourie (2001) como desintermediação da informação e se apresenta como uma tendência na sociedade, proporcionando autonomia e independência para os usuários da informação. Lopes (2005, p. 50) nos ensina que:

[...] esta ausência de contato usuário/bibliotecário denominou-se desintermediação da informação. O conceito desintermediação surgiu entre a década de 1960 e 1970. [...] os bancos foram os primeiros a adotar tal conceito quando passaram a oferecer seus serviços de forma que seus clientes o fizessem sem a intermediação humana. O processo de desintermediação nas bibliotecas se deu com maior intensidade com o surgimento da 
Internet.

Lopes (2005, p. 116) considera a desintermediação da informação "[...] como um fenômeno provocado pelo uso das TICs, que implica a inexistência de um intermediário entre o provedor da informação e seus usuários".

A maioria dos periódicos científicos, impressos ou eletrônicos, tem sua coleção descrita nos catálogos das bibliotecas (Online Public Access Catalogue-OPAC). Mesmo com a existência de uma lista on-line dos periódicos eletrônicos e de seus respectivos links, os usuários das bibliotecas exprimem a necessidade de acessar uma única lista, reunindo periódicos impressos e eletrônicos disponíveis dentro das bibliotecas de uma mesma instituição. A indisponibilidade de informação reunida e atualizada traz frustração ao usuário, em sua tentativa de localizar o periódico desejado (EWING, 2005).

Baseado em experiências internacionais, o presente trabalho apresenta propostas que facilitam o acesso à informação procurada (base de dados ou títulos de periódicos) por meio de sistemas de informação que remete o usuário diretamente à base de dados ou ao periódico desejado. Tais sistemas permitem a obtenção de respostas às suas reais necessidades, já no início do seu processo de busca de informações.

\title{
3 APRIMORANDO A INTERFACE COM O USUÁRIO PARA A ESCOLHA DE BASE DE DADOS OU PERIÓDICOS
}

Todo investigador tem como principal motivação a busca de respostas para questões dentro de seu campo de atuação. Para que os seus objetivos sejam atingidos, é fundamental haver a adequada vinculação de suas perguntas a situações de cunho real e compreensível.

\begin{abstract}
Atualmente, o acesso à informação eletrônica é um dos pontos altos das tecnologias de informação e comunicação aplicadas às consultas a bases de dados e bibliotecas. [...] Apesar de todas as vantagens que podemos obter na utilização das estratégias interativas, baseadas nas tecnologias da informação e comunicação, para apoiar a pesquisa acadêmica, no caso em estudo, concluímos que apenas as estratégias interativas que são categorizadas como estratégias de busca de informações, e que são consideradas de menor grau de interatividade são efetivamente utilizadas pelos professores/pesquisadores (PALMEIRA; TENÓRIO; LOPES, [2005], p. 1, grifo nosso).
\end{abstract}

Com cada vez mais recursos informacionais técnicos e científicos disponíveis na Internet, a falta de um processo de acesso a estes recursos de modo rápido, fácil e correto pode ser desanimador e consumir muito tempo de pesquisa. Diversos web sites são mudados sem que estas alterações sejam divulgadas, provedores de bases de dados adicionam e excluem revistas científicas sem aviso prévio e os usuários esperam ter Enc. Bibli: R. Eletr. Bibliotecon. Ci. Inf., Florianópolis, n. 23, 1ºm. 2007. 
disponíveis serviços de informação em tempo integral. Um banco de dados encaminhando aos endereços precisos de sites na web pode simplificar o acesso aos recursos informacionais oferecidos pela biblioteca. Eles são de fácil manutenção e atualização e estabelecem uma maneira eficiente de eliminar erros sobre as múltiplas páginas da web, significando maiores rapidez e eficiência nas pesquisas (DAVIS; ROBBINS, 2003).

$\mathrm{Na}$ University of Florida, foram observadas semelhanças nos padrões de pesquisa dos usuários da biblioteca, estudantes das ciências sociais. Nesta observação foi constatado, por exemplo, que estes usuários perdem algum tempo distinguindo entre informação na $\underline{w e b}$ e informação distribuída via web, catálogos de bibliotecas e índices de periódicos. Então, foram feitas tentativas para desenvolver estratégias para resolver tal problema. Como exemplo destas estratégias experimentadas, a biblioteca produziu um localizador de bases de dados, onde os usuários podem, rapidamente, selecionar um assunto e receber uma lista recomendando as bases de dados disponíveis para a pesquisa. Em sua fase inicial, o projeto remetia os estudantes para uma lista de bases de dados multidisciplinares com muitos artigos em texto completo (FREEDMAN; PICCININO, 2002).

No Dowling College, em Oakdale, New York, foram desenvolvidos o Journal Locator e o Database Locator. O Journal Locator ${ }^{1}$ providencia uma lista com todos os pontos de acesso que a biblioteca possui a um título de periódico, esteja ele em uma base de dados eletrônica ou fisicamente, na biblioteca. O Database Locator ${ }^{2}$ fornece uma lista com pontos de acesso as bases de dados, iniciando a pesquisa a partir dos assuntos indexados por elas. Estes localizadores são bases de dados dinâmicas, que promovem uma pesquisa precisa e atualizada em índices, criados com recursos interativos e que podem ser manipulados (DAVIS; ROBBINS, 2003).

Mas, na prática, como isto é feito? Algumas experiências ilustram como funcionam estas ferramentas de localização. Aqui serão brevemente descritas quatro das muitas propostas utilizadas nos Estados Unidos da América. Todas estas têm, como fator comum, o uso de bases de dados/interfaces com conteúdo dinâmico, que permitem localizar com facilidade diferentes tipos de informações, tais como: quais bases de dados indexam o assunto procurado, onde encontrar determinado título de periódico fisicamente, na biblioteca, ou em uma base de dados na Internet, paga ou gratuita, com texto completo ou somente com o resumo.

Até então era utilizado o $H T M L$, que é estático e dificulta a atualização e manutenção de bases de dados eletrônicas, como bem explica Davidson:

Web sites estáticos, por exemplo, apresentam sérios problemas

$1 \underline{\mathrm{http}: / / w w w . d o w l i n g . e d u / l i b r a r y / j o u r n a l d b / j n n l . s h t m l}$

2 http://www.dowling.edu/library/database/newtest/newdirectorypage.shtm

Enc. Bibli: R. Eletr. Bibliotecon. Ci. Inf., Florianópolis, n. 23, 1º sem. 2007. 
quando agrupados por disciplinas. Com um web site dinâmico, a centralização dos dados garante que a atualização seja feita apenas uma vez. Todo o sistema torna-se mais maleável e fácil de administrar, além de a base de dados que é orientada ao web site também permitir customização (DAVIDSON, 2001, tradução nossa).

Em 2001, a University of Arkansas apresentou sua solução ${ }^{3}$ utilizando o programa Microsoft Access, aliado à linguagem de Script ASP (Active Server Pages). Este modelo proporcionou muitas melhorias ao sistema, tais como as de administração e de manutenção centralizada dos dados, além de proporcionar a customização da interface de acordo com as necessidades da biblioteca. A centralização dos dados passa a ser crucial, pois isto demandaria, por exemplo, apenas uma atualização, que se refletiria em todos os links que estão relacionados a um título. Uma base de dados com uma lista dinâmica também é fácil de ser utilizada após sua implantação, pois ela não exige um conhecimento profundo de $H T M L$ ou de programação.

Davidson (2001) menciona que o processo de migração de uma base de dados "estática" para outra, "dinâmica", é custoso em termos de tempo e de trabalho, mas que os inúmeros benefícios decorrentes desta migração compensam os investimentos feitos. Ele enfatiza que, antes de tudo, é necessário pensar quais informações deverão ser selecionadas para integrar a base de dados.

O Dowling College, apresentou, em 2002, o Database Locator e o Journal Locator, mencionados anteriormente, usando, como softwares para criar a sua base de dados dinâmica, o Webserver - IIS (Internet Information Server), Microsoft Access e ASP como o Middleware.

O terceiro exemplo vem da East Carolina University, onde era necessário acessar quatro web sites diferentes para saber se a biblioteca possuía ou não determinado título de periódico (SHOUSE; CRIMI; LEWIS, 2001). A meta era facilitar, para os usuários, a localização dos periódicos eletrônicos ou impressos. Atualmente, a busca é feita através do título completo, palavra-chave do título ou do assunto. Esta melhoria decorreu da criação do E-Journal Locator ${ }^{5}$, que é uma base de dados desenvolvida em Microsoft Access, posteriormente aliada ao Cold Fusion, para torná-la acessível via web. A utilização do Microsoft Access foi importante, na fase inicial da implantação desta ferramenta de busca. Porém, a longo prazo, o uso de servidores do tipo $S Q L$ garantiu a

3 http://libinfo.uark.edu/eresources/subjects.asp

4 Deve ser observado que o ASP é um componente do Microsoft IIS. Para uso em outros servidores de web, é necessária a compra de outro software, como o Sun ONE Active Server Pages, que antes era conhecido como Chili!Soft ou iASP. (DAVIS, ROBBINS, 2002).

5 http://personal.ecu.edu/tedescol/EJournal.htm

Enc. Bibli: R. Eletr. Bibliotecon. Ci. Inf., Florianópolis, n. 23, 1ºm. 2007. 
estabilidade e a condição para a atualização simultânea da base e do acesso à consulta pelos usuários. O resultado da busca traz uma lista com os títulos dos periódicos, o link para o site de seu fornecedor e seu período de abrangência.

Em 2002, as bibliotecas do Massachusetts Institute of Technology (MIT), apresentaram a sua solução para os referidos problemas com o VERA (Virtual Electronic Resource Access). Através do $V E R A^{6}$, se pode recuperar a informação apresentada em uma lista alfabética de títulos, palavras-chave ou assuntos, tanto para as bases de dados quanto para os periódicos eletrônicos. Atualmente, a busca permite recuperação de dados classificados em até 75 assuntos diferentes. $O$ resultado fornece o título completo do periódico ou da base de dados, e direciona o usuário, através de um link, para a página eletrônica deste periódico ou base de dados. Estes resultados também informam a abrangência da base de dados, dos exemplares ou edições, etc., o formato e o acesso se via web, CD-ROM, se há restrições ao uso - somente para usuários licenciados junto ao MIT ou gratuito, se o título está disponível fora do campus, entre outras informações. O catálogo $O P A C$ também indica quais os periódicos, se impressos ou eletrônicos, e quais as bases de dados que estão disponíveis ao usuário. Cada resultado apresenta a localização do respectivo item na biblioteca, ou o link para o seu endereço eletrônico. $\mathrm{O}$ VERA serve como um complemento de informações ao citado catálogo.

As bibliotecas do MIT também disponibilizam um link para "Bases de Dados Gerais" e um link para os "Guias de Assunto", que são páginas da Internet, selecionadas pelos bibliotecários, mas que não estão listados no VERA ou no Barton ${ }^{7}$.

O VERA foi construído usando o programa FileMaker Pro. É administrado por um bibliotecário e por um grupo de seis pessoas, que representam um subgrupo de mais 30 outras pessoas selecionadoras de assuntos. Atualmente, as bibliotecas do MIT utilizam o Sistema Gerenciador de Bibliotecas da Ex Libris e estão estudando a possibilidade de migrar os dados do VERA para o Verde, outra ferramenta disponível no Ex Libris, como ilustra a Figura 1.

6 http://river.mit.edu/mitlibweb/FMPro?-db=RS_Items.fp5\&-Lay=web\&-format=ro_search.htm\&findany

7 nome dado ao catálogo $O P A C$ no $M I T$

Enc. Bibli: R. Eletr. Bibliotecon. Ci. Inf., Florianópolis, n. 23, 1º sem. 2007. 


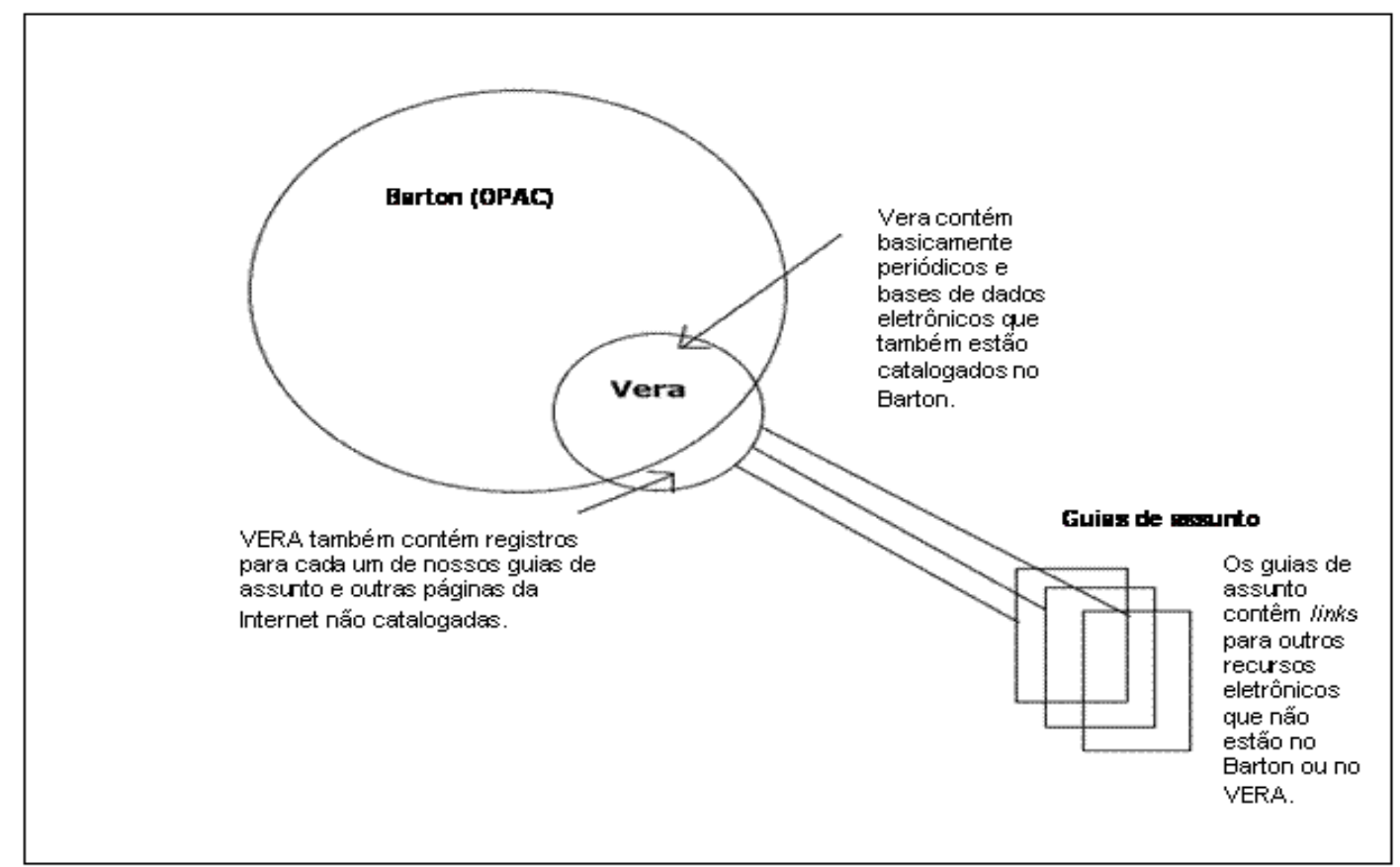

Figura 1 - Representação da proposta configuração da interface VERA.

Fonte: Adaptado e traduzido de Hennig (2002).

\section{APRIMORANDO A INTERFACE COM O USUÁRIO PARA A ESCOLHA DE BASE DE DADOS OU PERIÓDICOS NA UFRGS}

Atualmente, grande parte da coleção de bases de dados e de periódicos da Universidade Federal do Rio Grande do Sul (UFRGS), bem como das bibliotecas das demais Instituições de Ensino Superior (IES) do país, está disponível no Portal.periodicos da Capes. Pesquisa recente demonstra a limitação encontrada pelo usuário no uso da

[...] ferramenta de busca disponibilizada pelo portal da CAPES, [que] realiza pesquisa somente através de palavras e não através do contexto no qual a palavra está inserida, o que torna o acesso à informação menos eficiente. [...] Concluímos que o processo de consulta não é simples, ou seja, é necessário que o usuário esteja previamente capacitado para utilizar a ferramenta, como o próprio portal orienta, e também que este usuário tenha conhecimento dos assuntos, autores e áreas a serem consultados para sua pesquisa (PALMEIRA; TENÓRIO; LOPES, [2005], p. 19).

A página da Biblioteca Central da UFRGS disponível na Internet e o Periódicos da CAPES ilustram como esta diversidade de bases de dados, de recursos eletrônicos gratuitos e de vários fornecedores deixam o usuário confuso.

Segundo Walker:

Enc. Bibli: R. Eletr. Bibliotecon. Ci. Inf., Florianópolis, n. 23, 1ºm. 2007. 
[...] a grande maioria das bibliotecas universitárias tem meia dúzia ou mais de subsistemas locais, além do catálogo, geralmente de vários fornecedores, assim como acesso a centenas de bases de dados remotas, muitas das quais têm sérios problemas de interface. $\mathrm{O}$ que os usuários precisam não é de um $O P A C$ com uma interface melhor. Eles precisam é de uma interface simples, intuitiva, que integre todos os nossos sistemas. Softwares comerciais simplesmente não podem criar isso para nós. O único meio em que as bibliotecas podem oferecer esta união é através de APIs [Application Program Interface]. APIs permitem construir um único sistema, com uma interface simples, focando nos objetivos do usuário, que é simplesmente o de recuperar os dados disponíveis nos vários sistemas e bases de dados da biblioteca. Nós podemos começar a construir este sistema agora. Alguns, inclusive, já o fazem (WALKER apud DURRANCEAU, 2005, tradução nossa, grifo nosso).

A Biblioteca Central da Universidade de Brasília $(\mathrm{UnB})$ possui um recurso simplificado de busca a títulos de periódicos, que é denominado de "Busca Periódicos OnLine", onde é possível realizar, em uma busca por palavras do título, a recuperação do título do periódico, do período de abrangência do mesmo e da base de dados onde tal periódico pode ser encontrado.

Existem algumas limitações na proposta da UnB. Por um lado, ao ser feito o acesso ao link do título do periódico, o sistema responde com a página da base de dados como um todo, e deve ser repetida a pesquisa pelo título desejado, para finalmente se obter o acesso à revista. Por outro, este resultado de busca não mostra ao pesquisador a possível existência de uma coleção física, na biblioteca, do título procurado.

Atualmente, além de repetir a estratégia de busca várias vezes para se fazer uma pesquisa, deve ser conhecido, de antemão, o periódico desejado ou as bases de dados que indexam tais assuntos e títulos de periódicos, se estas bases apresentam o texto completo ou apenas o resumo, e qual é o editor ou o fornecedor responsável pela revista ou base de dados.

Para aprimorar a elaboração de uma interface mais adequada para a escolha de uma base de dados e de periódicos, é aqui feita a proposta de apresentação, aos usuários, de uma forma de pesquisar mais simples e intuitiva. Deve ser permitido que estes saibam: (a) que bases de dados estão disponíveis na biblioteca; (b) qual(ais) base(s) de dados eles deveriam consultar sobre um assunto específico; (c) em que base(s) de dados eles poderiam encontrar o título do periódico desejado; (d) qual o período de cobertura da base de dados que eles pretendem consultar; (e) se o periódico é indexado em mais de uma base de dados; (f) se o periódico procurado encontra-se disponível em texto completo ou se somente pode ser resgatado o seu resumo; $(\mathrm{g})$ se o arquivo deste artigo encontra-se disponível para download; (h) se existe a versão impressa deste artigo na

8 http://www.bce.unb.br/

Enc. Bibli: R. Eletr. Bibliotecon. Ci. Inf., Florianópolis, n. 23, 1º sem. 2007. 
biblioteca; e (i) quais são as bases de dados e periódicos eletrônicos disponíveis para acesso gratuito via Internet. Toda esta informação poderia ser unificada em uma operação.

Através dos casos relatados, foram apresentados alguns dos problemas e propostas de soluções encontradas para facilitar a recuperação de informações através de interfaces amigáveis.

Os softwares utilizados nestas propostas são conhecidos. Talvez, na UFRGS, visando à redução de custos sejam necessárias algumas adaptações para o uso de softwares livres, tais como o MySQL ou PHP. Estudos indicam a existência de iniciativas - tais como a criação de repositórios digitais temáticos - que garantam a interoperabilidade através do uso de softwares com código aberto (KURAMOTO, 2005), que viabilizariam a proposta apresentada no presente trabalho. Esta certamente seria a proposta de menor custo e a mais trabalhosa, porém o resultado tenderia a ser uma interface totalmente customizada às necessidades de nossos usuários.

Por outro lado, a UFRGS utiliza o mesmo programa gerenciador de bibliotecas do MIT - o Aleph/Ex Libris - que comercializa o portal Metalib sendo esta a segunda alternativa proposta. É possível vincular em busca única tanto ao $O P A C$, que fornece a localização do exemplar físico na biblioteca setorial, quanto ao Portal.periódicos. ou Internet, que exibe a localização eletrônica disponível para o periódico ou base de dados pesquisada. Esta representaria a alternativa de maior custo, contudo teríamos o suporte de uma grande empresa especializada neste tipo de software e com larga experiência no uso desta interface em grandes universidades espalhadas pelo mundo.

O início de tal proposta de aprimoramento de interface, seja na alternativa de software livre ou software comercial, deveria ter início com a constituição de um grupo multidisciplinar de estudo (composto por bibliotecários, analistas de sistemas, pesquisadores e estudantes), para listar todas as necessidades dos usuários. Depois de analisar as opções existentes no mercado e as geradas localmente, deve ser tomada a decisão pela escolha de uma ferramenta de busca e recuperação de informações indexadas que atenda às necessidades locais detectadas, levando em conta as vantagens econômicas e a excelência de prestação de serviços aos nossos usuários.

"Em um mundo Googled, Ipoded e Blackberried, todas as bibliotecas precisam repensar sobre como oferecer o seu conteúdo aos usuários - e o conteúdo será predominantemente digital" (DURANCEAU, 2005). A palavra-chave é: facilitar!

\section{REFERÊNCIAS}

CENDÓN, B. V. Serviços de indexação e resumo. In: CAMPELLO, B. S.; CENDÓN, B. V.; KREMER, J. M. (Orgs.). Fontes de informação para pesquisadores e profissionais. Belo Horizonte: Editora UFMG, 2000. p. 217-248. 
DAVIDSON, B. H. Database driven. Dynamic content delivery: providing and managing access to online resources using Microsoft Access and Active Server Pages. OCLC Systems and Services, v.17, n. 1, p. 34-42, 2001. Disponível em: http://www.emeraldinsight.com/Insight/ViewContentServlet?Filename=Published/Emer aldFullTextArticle/Articles/1640170104.html. Acesso em: 02 jun. 2006.

DAVIS, F. C.; ROBBINS, L. P. Potentials of dynamic database-driven web sites. In: ACRL National Conference, (11. : 2003 : Charlotte). [Proceedings ...] Disponível em: http://www.ala.org/ala/acrl/acrlevents/pope-robbins.pdf. Acesso em: 01 jun 2006.

DURANCEAU, E. From Vera to Verde? The MIT libraries look at leaving a local ERM system behind. In: NELA Annual Conference, 2005. [Proceedings...] Disponível em: http://64.233.161.104/search?q=cache:75B5jiCPBUkJ:www.nelib.org/nets1/pastprogra ms/ERM\%2520Duranceau.ppt+DURANCEAU,+Ellen.+From+Vera+to+Verde\%3F\&hl $=\mathrm{pt}-\mathrm{BR} \& \mathrm{gl}=\mathrm{br} \& \mathrm{ct}=\mathrm{clnk} \& \mathrm{~cd}=1$ Acesso em: 15 jun 2006.

EWING, C. C. Creating a web-accessible combined print and electronic journal listing. Journal of Electronic Resources in Medical Libraries, Binghamton, NY, v. 2, n. 2, 2005. Disponível em: http://www.haworthpress.com/web/JERML. Acesso em: 01 jun 2006.

FOURIE, I. Debemos tomarnos en serio la desintermediación? Anales de Documentación: revista de biblioteconomia e documentación, Murcia v. 4, p. 267-282, 2001. Disponível em: http://www.um.es/fccd/anales/ad04/ad0400.html. Acesso em: 12 fev. 2007.

FREEDMAN, T.; PICCININO, R. College Science Librarians Discussion Group. STS Signal, Chicago, v. 17, n. 2, Fall 2002. Disponível em: http://www.ala.org/ala/acrl/aboutacrl/acrlsections/sciencetech/stspublications/fall02.pdf Acesso em: 01 jun 2006.

HENNIG, N. Improving access to e-journals and databases at the MIT Libraries: building a database-backed web site called "VERA". Serials Librarian, v. 41, n. 34, p.227-254, 2002. Disponível em: http://www.hennigweb.com/publications/vera.html\#10. Acesso em: 02 jun. 2006.

KURAMOTO,H. Os Open Archives e as política públicas para a informação científica. In: CONGRESSO BRASILEIRO DE CIÊNCIAS DA COMUNICAÇÃO, 28., 2005, Rio de Janeiro. Anais... São Paulo: Intercom, 2005. Disponível em: http://reposcom.portcom.intercom.org.br/bitstream/1904/18468/1/R4597-1.pdf. Acesso em: 14 jul. 2006.

LOPES, M. I. A Internet e a busca da informação em comunidades científicas: um estudo focado nos pesquisadores da UFSC. Dissertação - (Mestrado) - Universidade Federal de Santa Catarina, Programa de Pós-Graduação em Ciência da Informação, 2005. Florianópolis, 2005. 184f. 
MEADOWS, A. J. A comunicação científica. Brasília: Briquet de Lemos, 1999.

MUELLER, S. P. M. O periódico científico. In: CAMPELLO, B. S.; CENDÓN, B. V.; KREMER, J. M. (Orgs.). Fontes de informação para pesquisadores e profissionais. Belo Horizonte: Editora UFMG, 2000. p. 73-96.

PAIM, I.; NEHMY, R. M. Q. Questões sobre a avaliação da informação: uma abordagem inspirada em Giddens. Perspectivas em Ciência da Informação, Belo Horizonte, v. 3, n. 2, p. 81-95, jul./dez.1998.

PALMEIRA, M. F.; TENÓRIO, R. M.; LOPES, U. M. O uso das ferramentas interativas baseadas nas tecnologias da informação e comunicação na pósgraduação. [2005]. Disponível em: http://www.gepicc.ufba.br/enlepicc/pdf/UacaiLopes.pdf. Acesso em: 01 jun 2006.

SHOUSE, D. L.; CRIMI, N.; LEWIS, J. S. Managing journals: one library's experience. Library Hi Tech, Bradford, UK, v. 19, n. 2, p.150-155, 2001. Disponível em: http://www.emeraldinsight.com/Insight/viewContentItem.do?contentType=Article\&hd Action=lnkpdf\&contentId=861269. Acesso em: 01 jun. 2006.

TEIXEIRA, C. M. de S.; SCHIEL, U.. A Internet e seu impacto nos processos de recuperação da informação. Ciência da Informação, Brasília, DF, v. 26, n. 1, jan./abr. 1997. Disponível em: http://www.scielo.br/scielo.php?script $=$ sci arttext\&pid=S0100$19651997000100009 \& \operatorname{lng}=$ pt\&nrm=iso Acesso em 01 jun 2006.

\begin{abstract}
Information and communication technologies had produced deep transformations in the way publications and other kinds of scientific information flow in academic environments. With the development and popularization of these technologies, many documents are now available in both printed and electronic format. As the number of available documents increases, new questions arise: how can one improve the user interface for better choosing a database? Which databases contain the desired periodic? Is a given periodic heading indexed by more than one database? Is the complete periodic available or only its summary? Which database must be queried on a specific subject? Librarians of North American universities had proposed some solutions to these questions by essentially trying to simplify and optimize the search in the library catalogues (Online Public Access Catalogue - OPAC). The present work proposes the application of the North American idea in the Universidade Federal do Rio Grande do Sul (UFRGS) library system. More specifically, we would like to provide a high-level interface for automatically combining the user's searches simultaneously to the UFRGS library system, to the entire set of databases available through the Portal.periodicos from CAPES, and to the free databases available on the Internet.
\end{abstract}

KEYWORDS: University Library. Information retrieval. Bibliographical research.

\title{
GLOSSÁRIO
}

Enc. Bibli: R. Eletr. Bibliotecon. Ci. Inf., Florianópolis, n. 23, 1ºm. 2007. 
Active Server Pages - capacita a criação de páginas dinâmicas na web, usando HTML, scripts e ActiveX. Geralmente essas páginas interagem com bases de dados.

APIs - ver Application Program Interface.

Application Program Interface - Conjunto de ferramentas de programação que predefinem funções e rotinas utilizadas para permitir que um programa se comunique com outro, agrupando o material de programação comum em blocos.

ASP - ver Active Server Pages

BlackBerry - aparelho portátil, sem fio, com recursos de e-mail, telefone, editor de texto e busca na internet.

CD-ROM - ver Compact Disc Read Only Memory.

Chili!Soft ASP - linguagem de programação para computadores, usada principalmente para a World Wide Web. Componente desenvolvido nos Estados Unidos da América, pela

Chili!Soft (http://developers.sun.com/asp/whitepapers/chilisoft asp_usersguide.pdf) que permite a execução de páginas ASP em ambiente Unix ou LinuxCold.

Compact Disc Read Only Memory - Disco plástico revestido por uma camada de metal, que permite o armazenamento e a leitura de dados utilizando-se da tecnologia óptica. Seu conteúdo é gravado apenas uma vez e não pode ser alterado.

Ex Libris - grupo mundial pioneiro na área de soluções de software para bibliotecas e centros de informação. O desenvolvimento do sistema ALEPH, por esta empresa, foi iniciado em 1980.

FileMaker Pro - é uma base de dados multi-plataforma da FileMaker, Inc. É reconhecida por ser uma base que combina potência à facilidade de uso e pela estreita ligação da base de dados e a interface gráfica, que permite que os usuários modifiquem a base de dados arrastando elementos novos nos layouts/screens/forms.

HTML - ver Hyper Text Markup Language.

Hyper Text Markup Language - linguagem de formatação mais utilizada nos documentos publicados na Internet, sendo capaz de dar formato a textos, imagens, sons e vídeos, e, principalmente, de vincular diferentes tipos de arquivos, por meio de links.

iASP - ver Chili!Soft ASP

Internet - Qualquer conjunto de redes de computadores ligadas entre si por roteadores e gateways.

Microsoft Access - um banco de dados relacional que permite o desenvolvimento rápido de aplicações que envolvem tanto a modelagem e estrutura de dados como 
também a interface a ser utilizada pelos usuários.

Middleware - software de interface que permite interação de diferentes aplicações de softwares, geralmente sobre diferentes plataformas de hardware e infra-estrutura, para troca de dados.

MySQL - é um sistema aberto de gerenciamento de bancos de dados relacionais, baseado em comandos SQL (Structured Query Language) ou linguagem estruturada para pesquisas.

Online Public Access Catalog - catálogo on line, versão automatizada do catálogo tradicional da biblioteca.

OPAC - ver Online Public Access Catalog.

PHP - um acrônimo recursivo para PHP: Hypertext Preprocessor. É uma linguagem de programação de computadores interpretada, livre e muito utilizada para gerar conteúdo dinâmico na World Wide Web.

SQL - é uma sintaxe usada para a definição e manipulação de dados em um banco de dados relacional. Desenvolvido pela IBM, em 1970, tornou-se um padrão industrial para linguagens de consulta, na maioria dos sistemas gerenciadores de bancos de dados relacionais.

VERA - ver Virtual Electronic Resource Access.

Verde - é um novo sistema que foi desenvolvido para complementar a suíte Ex Libris de ferramentas, que auxilia as bibliotecas e os centros de informação a gerenciar as fontes eletrônicas de informação durante todo o seu ciclo de vida, além de fornecer acesso a essas fontes.

Virtual Electronic Resource Access - Recursos eletrônicos de acesso virtual.

Web - Recurso ou serviço oferecido na Internet (rede mundial de computadores), e que consiste de um sistema distribuído de acesso a informações, as quais são apresentadas na forma de hipertexto, com elos entre documentos e outros objetos (menus, índices), localizados em pontos diversos da rede.

Webserver-IIS - Internet Information Server, o software para servidores da Microsoft operando nos sistemas Windows NT/2000.

Originais recebidos em 13/11/2006

Texto aprovado em 15/03/2007 